\title{
NOTE ON THE MOMENTS OF A BERNOULLI DISTRIBUTION*
}

\author{
BY A. T. CRAIG
}

If $p$ and $q=1-p$ denote, respectively, the probabilities of the success and the failure of an event in a single trial, then

$$
f(x)=\frac{n !}{x !(n-x) !} q^{n-x} p^{x}, \quad(x=0,1, \cdots, n),
$$

is the probability of exactly $x$ successes in $n$ trials provided $p$ is held constant throughout the $n$ trials. A frequency distribution whose relative frequencies are given in accord with this law of probability is sometimes called a Bernoulli distribution.

The moments (per unit frequency) of a frequency distribution have long been regarded as useful characteristics of the distribution. We shall denote the $s$ th moment about the vertical axis through the origin by $\mu_{s}^{\prime}$, while the corresponding moment about the arithmetic mean will be denoted by $\mu_{s}$. Thus, for the distribution given above, $\mu_{s}^{\prime}=\sum_{x=0}^{n} x^{8} f(x)$, and, since the arithmetic mean is $\mu_{1}^{\prime}, \mu_{s}=\sum_{x=0}^{n}\left(x-\mu_{1}^{\prime}\right)^{s} f(x)$. While it is easy to see that $\mu_{1}^{\prime}=n p$, it is not so easy to see what the results are for $s$ arbitrary. With respect to this problem Karl Pearson remarked in an editorial $t \dagger$ "A simple reduction formula for the moments of a binomial $(p+q)^{n}$ about its mean was sought in vain. After a good deal of energy had been spent, we believe that $\mu_{s}$, being the $s$ th moment about the mean,

$$
\mu_{s}=\left[\frac{d^{s}}{d x^{s}}\left(q e^{p x}+p e^{-q x}\right)^{n}\right]_{x=0},
$$

is, perhaps, the easiest expression for reaching these moment coefficients by successive differentiation." However, Romanovsky $\ddagger$ has derived the recursion formula

* Presented to the Society, December 27, 1933.

$\dagger$ Karl Pearson, Biometrika, vol. 12 (1918-1919), footnote, p. 270.

$\ddagger$ V. Romanovsky, Note on the moments of the binomial $(p+q)^{n}$ about its mean, Biometrika, vol. 15 (1923), pp. 410-412. See also Les Principes de la Statistique Mathématique, 1933, pp. 39-40 and pp. 320-321. 


$$
\mu_{s+1}=p q\left[n s \mu_{s-1}-\frac{d \mu_{s}}{d q}\right]
$$

for the moments about the mean. While Romanosvky's proof is very elegant, it may be of some interest to know that we can arrive at the recursion formula with considerably less effort. Also, by the same simple method, we find other recursion formulas for moments. From

$$
\mu_{s}^{\prime}=\sum_{x=0}^{n} x^{s} \frac{n !}{x !(n-x) !} q^{n-x} p^{x},
$$

we have

$$
\begin{aligned}
& \frac{d \mu_{s}^{\prime}}{d q}=\sum_{x=0}^{n} x^{s} \frac{n !}{x !(n-x) !}\left\{q^{n-x-1} p^{x}(n-x)-x q^{n-x} p^{x-1}\right\} \\
& =\frac{n}{q} \sum_{x=0}^{n} x^{s} \frac{n !}{x !(n-x) !} q^{n-x} p^{x}-\frac{1}{p q} \sum_{x=0}^{n} x^{s+1} \frac{n !}{x !(n-x) !} q^{n-x} p^{x},
\end{aligned}
$$

since $p=1-q$. Thus

$$
\frac{d \mu_{s}^{\prime}}{d q}=\frac{n}{q} \mu_{s}^{\prime}-\frac{1}{p q} \mu_{s+1}^{\prime}, \quad \text { and } \mu_{s+1}^{\prime}=p q\left[\frac{n}{q} \mu_{s}^{\prime}-\frac{d \mu_{s}^{\prime}}{d q}\right] .
$$

Also, from

$$
\mu_{s}=\sum_{x=0}^{n}(x-n p)^{s} \frac{n !}{x !(n-x) !} q^{n-x} p^{x},
$$

we have

$$
\begin{aligned}
\frac{d \mu_{s}}{d q}= & -\sum_{x=0}^{n} \frac{x(x-n p)^{s} n !}{x !(n-x) !} q^{n-x} p^{x-1} \\
& +\sum_{x=0}^{n} \frac{(x-n p)^{s} n !}{x !(n-x) !} q^{n-x-1} p^{x}(n-x) \\
& +\sum_{x=0}^{n} \frac{n s(x-n p)^{s-1} n !}{x !(n-x) !} q^{n-x} p^{x} \\
= & -\frac{1}{p q} \mu_{s+1}+n s \mu_{s-1} .
\end{aligned}
$$

Whence we have 


$$
\mu_{s+1}=p q\left[n s \mu_{s-1}-\frac{d \mu_{s}}{d q}\right]
$$

which is Romanovsky's formula.

It is interesting to observe that we may use the same method to find recursion formulas for the moments of the Poisson exponential $g(x)=\lambda^{x} e^{-\lambda} / x !$, where $\lambda>0, x=0,1, \cdots, n$, and $n$ is large. Thus, from

$$
\mu_{s}^{\prime}=\sum_{x=0}^{n} \frac{x^{s} \lambda^{x} e^{-\lambda}}{x !}
$$

we have

$$
\frac{d \mu_{s}^{\prime}}{d \lambda}=-\sum_{x=0}^{n} \frac{x^{s} \lambda^{x} e^{-\lambda}}{x !}+\frac{1}{\lambda} \sum_{x=0}^{n} \frac{x^{s+1} \lambda^{x} e^{-\lambda}}{x !}=-\mu_{s}^{\prime}+\frac{1}{\lambda} \mu_{s+1}^{\prime},
$$

and accordingly

$$
\mu_{s+1}^{\prime}=\lambda\left(\mu_{s}^{\prime}+\frac{d \mu_{s}^{\prime}}{d \lambda}\right)
$$

Also, from

$$
\mu_{s}=\sum_{x=0}^{n}(x-\lambda)^{s} \frac{\lambda^{x} e^{-\lambda}}{x !}
$$

we have

$$
\frac{d \mu_{s}}{d \lambda}=\frac{1}{\lambda} \sum_{x=0}^{n}(x-\lambda)^{s+1} \frac{\lambda^{x} e^{-\lambda}}{x !}-s \sum_{x=0}^{n}(x-\lambda)^{s-1} \frac{\lambda^{x} e^{-\lambda}}{x !} .
$$

Hence, we have

$$
\mu_{s+1}=\lambda\left(s \mu_{s-1}+\frac{d \mu_{s}}{d \lambda}\right) .
$$

The University of Iowa 